\title{
AUMENTO DE PRODUTIVIDADE NO LAMINADOR DE TIRAS A QUENTE: MODO DE OPERAÇÃO RM-NECK*
}

Thiago Vieira Lopes ${ }^{1}$

Rafael Nascimento Silvar ${ }^{2}$ Ricardo Lessa Correa ${ }^{3}$ Sidney Ferreira da Silva ${ }^{4}$ Fábio de Oliveira Araújo ${ }^{4}$ Arthur Guimarães Alves ${ }^{5}$

\section{Resumo}

O mercado siderúrgico atual encontra-se cada vez mais competitivo e exige das empresas esforços na diminuição do custo de produção para que seus produtos mantenham atratividade no cenário global. A incorporação crescente de produtos siderúrgicos oriundos da Ásia no mercado ocidental obriga as indústrias encontrarem alternativas de diminuição de custo de produção, as rotas para que tal objetivo seja alcançado geralmente estão ligadas ao aumento do rendimento metálico e diminuição no tempo de processamento dos produtos. O equipamento gargalo na linha de laminação a quente da CSN, é a cadeira reversível da laminação de desbaste, R2, e pequenas reduções de tempo no processamento de placas são responsáveis por incrementos elevados na produtividade da laminação. Visando ampliar a competitividade da CSN no cenário siderúrgico foi criada uma função, chamada RM-NECK, no modelo matemático do laminador que classifica todos os materiais gargalo desbaste por grau, espessura, largura, número de fornos de reaquecimento e comprimento de placa, permitindo assim, a diminuição no tempo de processamento na zona do VE2/R2. O estudo evoluiu para um verdadeiro trabalho em equipe, envolvendo especialistas da manutenção e operação e, consolidando anos de experiência no desenvolvimento de um algorítmo inteligente o suficiente para reduzir o tempo de processo observando os princípios da teoria das restrições.

Palavras-chave: Produtividade; Custo de produção; RMNECK.

\section{PRODUCTIVITY INCREASE IN THE HOT STRIP MILL: RM-NECK OPERATION MODES}

\author{
Abstract \\ The current steel market is increasingly competitive and it requires efforts from companies to \\ decrease the production costs so that products remain attractive in the global scenario. The \\ growing incorporation of steel products manufactured in Asia requires Brazilian steel makers \\ to find alternatives to decrease production cost. The routes to achieve such goal are generally \\ linked to increasing metal yield and decreasing processing time. The main bottleneck on CSN \\ hot rolling line is the reversing mill, R2, and even small reductions in processing time at the \\ mentioned stand are responsible for increasing considerably global hot strip mill production \\ rate. Aiming to increase the competitiveness of the CSN steel, it was created a function, called \\ RM-NECK, setted at the mathematical model of the rougher mill. RM-NECK identifies cases \\ in which the reversing mill will be the production bottleneck by observing strip and plate \\ dimensions, steel grade and number of reheating furnaces in operation. For the mentioned \\ cases, a specific function operates aiming to reduce processing time at VE2/R2 zone. The \\ study evolved to a true team work, involving maintenance and operation specialists who \\ consolidated years of experience in the development of an enough intelligent algorithm to \\ reduce the process time, observing the principles of the theory of constraint. \\ Keywords: Production rate; Production costs; RMNECK. \\ 1 UFF - Universidade Federal Fluminense, Engenheiro Metalurgista, Engenheiro de Operação Sr, \\ Laminação a Quente, Companhia Siderúrgica Nacional (CSN), Volta Redonda, Rio de Janeiro, Brasil. \\ 2 UBM - Universidade Barra Mansa, Eng. de Automação, Eng. de Operação Jr, Laminação a Quente, \\ CSN, Volta Redonda, RJ, Brasil. \\ UniFoa, Eng. Cívil, Eng. de Manutenção Sr. Laminação a Quente, CSN, Volta Redonda, RJ, Brasil. \\ UFF, Eng. Metalurgista, Eng. Especialista, Laminação a Quente, CSN, Volta Redonda, RJ, Brasil. \\ $1^{\circ}$ Tenente do Quadro de Oficiais Engenheiros, Força Aérea Brasileira, RJ, Brasil.
}




\section{INTRODUÇÃO}

O mercado siderúrgico brasileiro atualmente possui grandes dificuldades de crescimento devido à incorporação crescente de produtos originários do mercado asiático. Os preços de venda dos produtos importados muitas vezes estão muito próximos ou até mesmo inferiores aos produtos produzidos no mercado interno, este fato gera uma disputa acirrada pelo mercado. A fim de minimizar os efeitos gerados pela entrada de produtos importados no mercado, a indústria nacional está procurando maneiras de reduzir o custo de produção com o intuito de diminuir os preços de seus produtos e assim conseguir reverter o quadro atual da balança comercial.

Amplamente utilizada como estratégia de diminuição de custos de produção por um grande número de indústrias, o aumento da produtividade do processo sem degradação do maquinário e grandes investimentos vem tornando-se a opção com maior aceitação dentro do ambiente industrial.

Todos os produtos laminados presentes no portfólio da CSN, Companhia Siderúrgica Nacional, são processados inicialmente no laminador de tiras a quente, fato que garante a grande importância deste equipamento. A laminação a quente pode ser dividida em quatro grandes células: Fornos de Reaquecimento de Placa, Laminação de Desbaste, Laminação de Acabamento e Bobinadoras. O equipamento gargalo do laminador a quente, ou seja, aquele que possui maior tempo de processo para a maioria dos materiais é a cadeira R2. A cadeira R2 é um laminador horizontal quadruo reversível que está localizado na célula da laminação de Desbaste e desempenha papel fundamental na laminação a quente.

A fim de reduzir os custos globais de transformação das bobinas a quente, a cadeira R2 aparece como uma janela de oportunidades. A redução de poucos segundos por placa no processamento deste equipamento pode gerar um aumento de produtividade no laminador de tiras a quente e consequentemente reduzir os custos de transformação das bobinas a quente.

\subsection{Teoria das Restrições - TOC}

A Teoria das Restrições (theory of constraints) aborda uma filosofia que oferece meios de identificar e tratar pontos específicos que impedem a empresa de atingir suas metas. A teoria das restrições foi publicada em 1984 pelo físico israelense Eliyahu Goldratt através do livro The Goal (A Meta) e desde então vem sendo amplamente utilizada em diversos setores produtivos. [1]

De acordo com a teoria, o processo de laminação ilustrado na 1 a seguir, o qual temse uma produção dividida em três subprocessos: fornos de aquecimento; laminadores desbastadores; e laminadores de acabamento, com o tempo de processamento de 76, 72 e 85 segundos, respectivamente, têm como restrição os laminadores de acabamento, por ser o subprocesso onde gasta-se um tempo de processamento relativamente maior que os demais. 


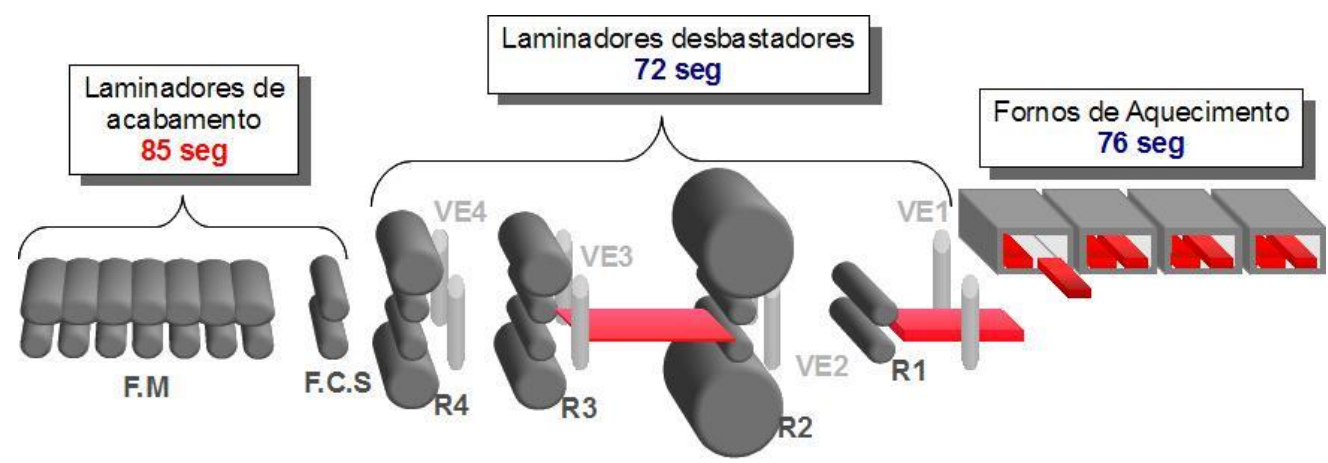

Figura 1: Análise de gargalo de um processo de laminação, cenário 1. O laminador de acabamento é a etapa do processo que possui maior tempo de processamento, sendo assim o ponto gargalo da linha.[2]

No entanto, trabalhando, somente, na redução do tempo gasto na laminação de acabamento, trazendo-a para 74 segundos, a restrição passa a ser os fornos de aquecimento, com tempo de 76 segundos, conforme a Figura 2.

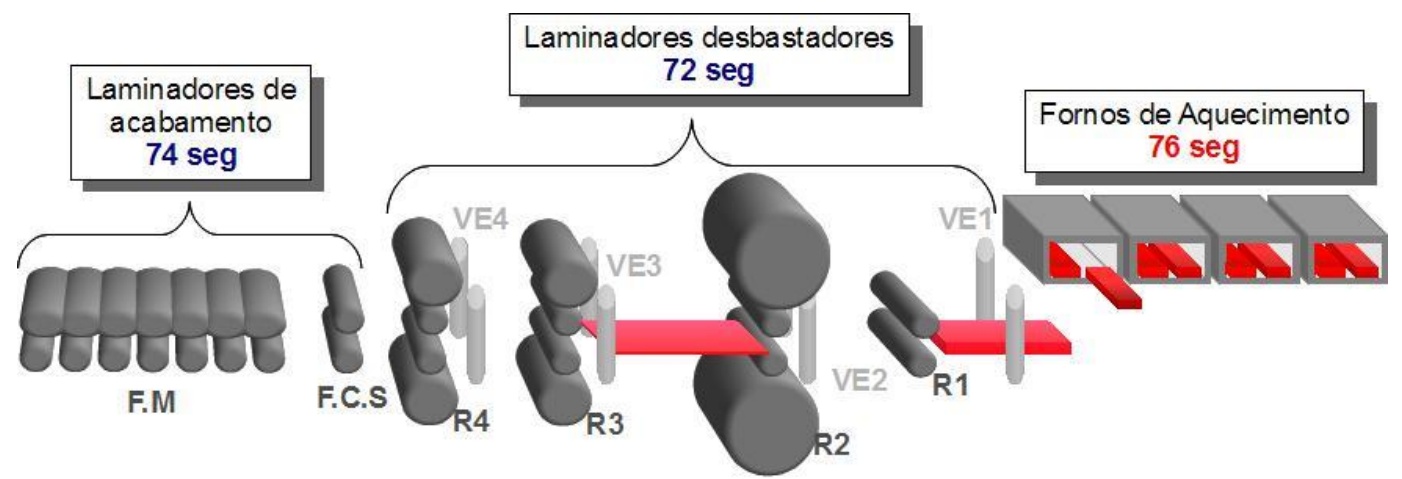

Figura 2: Análise de gargalo de um processo de laminação, cenário 2. O laminador de acabamento após redução de tempo de processo deixa de ser o equipamento gargalo.[2]

Assim, vê-se que conforme cita a Teoria, o tempo de produção gasto em um subprocesso deve ser sensivelmente equiparado ao tempo gasto nos demais.

\subsection{Avaliação do Gargalo}

A decisão de aplicar melhorias no funcionamento da cadeira R2 se deu após avaliação do desempenho geral da linha, procurando identificar o ponto de maior incidência de gargalo, ou seja, onde o processo apresentava maior índice de restrição. Esta análise foi feita com base na teoria das restrições que diz que o resultado esperado somente será alcançado mediante o bom desempenho de todos os elementos envolvidos em um determinado processo. A linha de produção será tão produtiva quanto seu setor que apresenta a maior restrição, sendo assim, levando em conta que as restrições de um sistema impactarão diretamente no resultado, devem-se intensificar os esforços no sentido de atuar nas áreas em que as melhorias resultarão em uma evolução global do processo. 


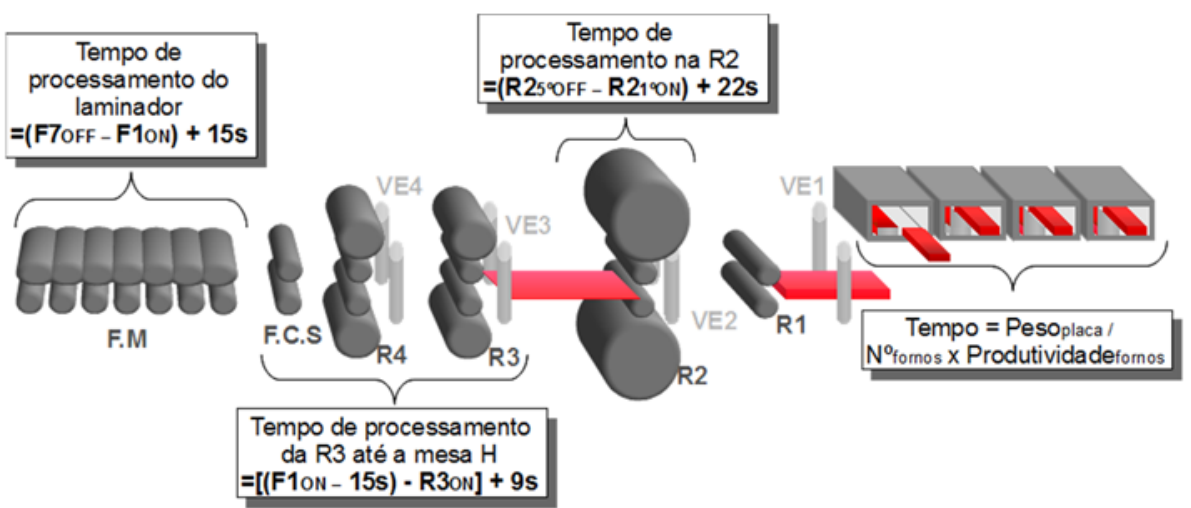

Figura 3: Método de avaliação de gargalo de produção pela comparação dos tempos dos setores. [2]

Conforme a Figura 3, através das equações que relacionam o tempo de processo com o momento em que cada tira pode ocupar a posição seguinte na linha de produção, pode-se mapear o tempo de processamento das diferentes dimensões de tiras laminadas para cada setor, separando-as em espessura e largura, conforme a Tabela 1.

Tabela 1: Setor gargalo com relação de espessura e largura.

\begin{tabular}{|c|c|c|c|c|c|c|c|c|}
\hline 2013 & & & & Largura & & & & \\
\hline Espessura & $<800 \mathrm{~mm}$ & $800-950 \mathrm{~mm}$ & $950-1050 \mathrm{~mm}$ & $1050-1150 \mathrm{~mm}$ & $1150-1250 \mathrm{~mm}$ & $1250-1350 \mathrm{~mm}$ & $1350-1450 \mathrm{~mm}$ & $>1450 \mathrm{~mm}$ \\
\hline $1,4-1,65 \mathrm{~mm}$ & Acabamento & Acabamento & Acabamento & Acabamento & Acabamento & Acabamento & Acabamento & Forno \\
\hline $1,65-1,89 \mathrm{~mm}$ & Acabamento & Acabamento & Acabamento & Acabamento & Acabamento & Acabamento & Acabamento & Forno \\
\hline $1,89-1,99 \mathrm{~mm}$ & Acabamento & Acabamento & Acabamento & Acabamento & Acabamento & Acabamento & Acabamento & Forno \\
\hline $1,99-2,20 \mathrm{~mm}$ & Acabamento & Acabamento & Acabamento & \begin{tabular}{|l|} 
Acabamento \\
\end{tabular} & Acabamento & Acabamento & Acabamento & Forno \\
\hline $2,20-2,50 \mathrm{~mm}$ & Desbaste /Acabamento & Desbaste /Acabamento & Desbaste /Acabamento & Desbaste /Acabamento & Desbaste /Acabamento & Desbaste /Acabamento & Desbaste /Acabamento & Forno \\
\hline $2,50-2,70 \mathrm{~mm}$ & Desbaste /Acabamento & Desbaste /Acabamento & Desbaste /Acabamento & Desbaste /Acabamento & Desbaste /Acabamento & Desbaste /Acabamento & Desbaste /Acabamento & Forno \\
\hline $2,70-2,90 \mathrm{~mm}$ & \begin{tabular}{|c|} 
Desbaste \\
\end{tabular} & \begin{tabular}{|c|} 
Desbaste \\
\end{tabular} & \begin{tabular}{|c|} 
Desbaste \\
\end{tabular} & \begin{tabular}{|c|} 
Desbaste \\
\end{tabular} & \begin{tabular}{|c|} 
Desbaste \\
\end{tabular} & \begin{tabular}{|c|} 
Desbaste \\
\end{tabular} & \begin{tabular}{|c|} 
Desbaste \\
\end{tabular} & Forno \\
\hline $2,90-3,20 \mathrm{~mm}$ & Desbaste & Desbaste & Desbaste & Desbaste & Desbaste & Desbaste & Desbaste & Forno \\
\hline $3,20-3,60 \mathrm{~mm}$ & Desbaste & Desbaste & Desbaste & Desbaste & Desbaste & Desbaste & Desbaste & Forno \\
\hline $3,60-4,10 \mathrm{~mm}$ & Desbaste & Desbaste & Desbaste & Desbaste & Desbaste & Desbaste & Desbaste & Forno \\
\hline $4,10-4,50 \mathrm{~mm}$ & Desbaste & Desbaste & Desbaste & Desbaste & Desbaste & Desbaste & Desbaste & Forno \\
\hline $4,50-4,75 \mathrm{~mm}$ & Desbaste & Desbaste & Desbaste & Desbaste & Desbaste & Desbaste & Desbaste & Forno \\
\hline $4,75-5,07 \mathrm{~mm}$ & Desbaste & Desbaste & Desbaste & Desbaste & Desbaste & Desbaste & Desbaste & Forno \\
\hline $5,07-5,60 \mathrm{~mm}$ & Desbaste & Desbaste & Desbaste & Desbaste & Desbaste & Desbaste & Desbaste & Forno \\
\hline $5,60-6,40 \mathrm{~mm}$ & Desbaste & Desbaste & Desbaste & Desbaste & Desbaste & Desbaste & Desbaste & Forno \\
\hline $6,40-7,90 \mathrm{~mm}$ & Desbaste & Desbaste & Desbaste & Desbaste & Desbaste & Desbaste & Desbaste & Forno \\
\hline $7,90-9,10 \mathrm{~mm}$ & Desbaste & Desbaste & Desbaste & Desbaste & Desbaste & Desbaste & Desbaste & Forno \\
\hline $9,10-10 \mathrm{~mm}$ & Desbaste & Desbaste & Desbaste & Desbaste & Desbaste & Desbaste & Desbaste & Forno \\
\hline$>10 \mathrm{~mm}$ & Desbaste & Desbaste & Desbaste & Desbaste & Desbaste & Desbaste & Desbaste & Forno \\
\hline
\end{tabular}

Observou-se na análise dos tempos de processamento que os três setores do processo podem se tornar o gargalo de produção de acordo com as diferentes dimensões laminadas. No entanto, essa propriedade situou-se majoritariamente no setor de desbaste, especificamente na laminação das tiras de espessuras acima de 2,70 $\mathrm{mm}$ e com largura abaixo de $1450 \mathrm{~mm}$.

A avaliação dos materiais processados em 2013 mostrou a distribuição dos gargalos conforme mostrado no gráfico da Figura 4. 


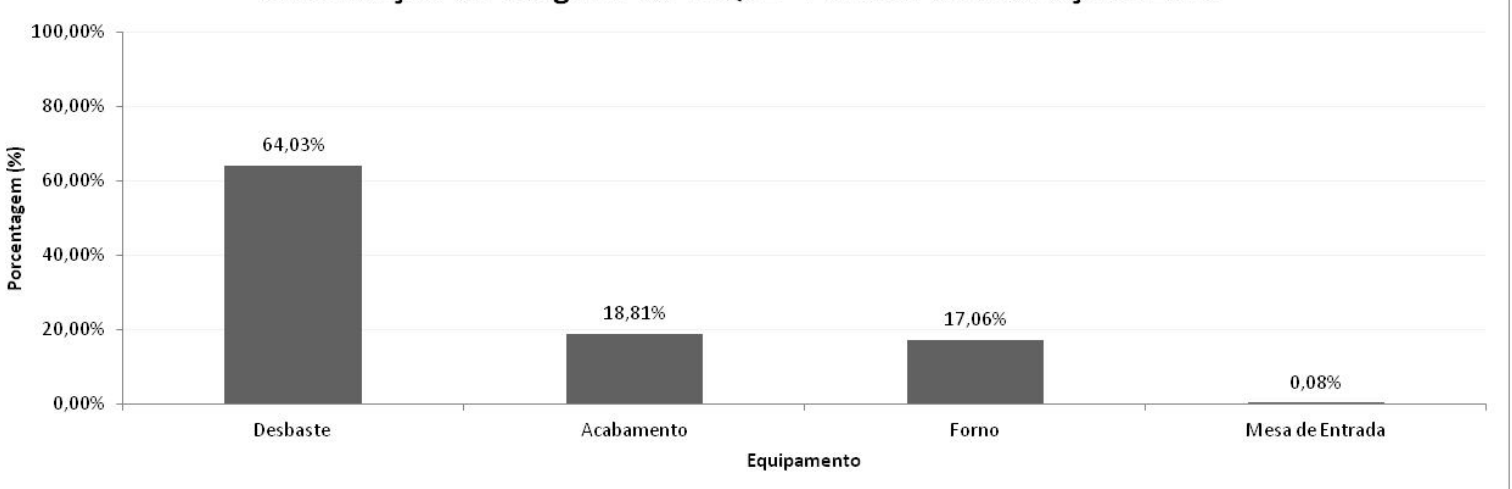

Figura 4: Distribuição dos gargalos da linha do laminador de tiras a quente no ano de 2013.

\subsection{Avaliação do IRMS}

O IRMS se trata da média quadrática dos valores de corrente alcançadas pelo motor, sendo assim, para garantir um regime de trabalho constante sem que o IRMS ultrapasse a meta estipulada, é necessário respeitar os tempos de trabalho e de espera do motor, desse modo a máquina opera sem que haja precocidade no desgaste de suas partes internas bem como risco de desarme e consequentemente interrupção do processo.

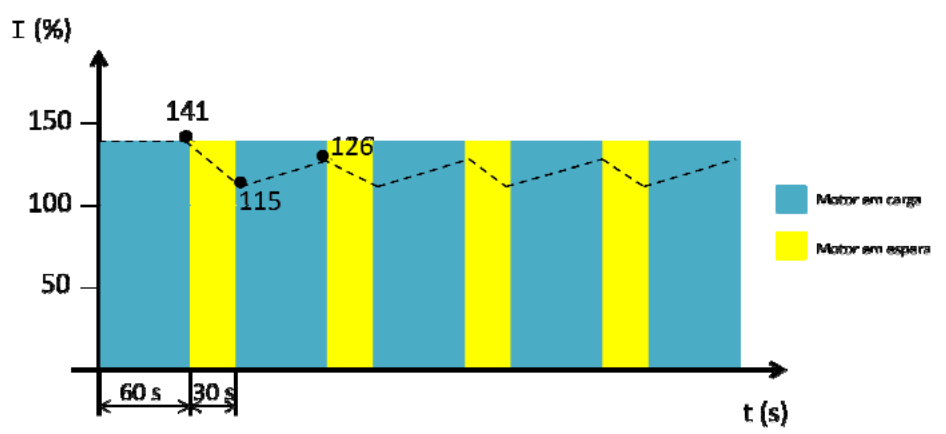

Figura 5: Gráfico hipotético para representação da variação IRMS em um motor. Adaptada de [3]

No caso dos motores principais da cadeira R2, o valor limite de IRMS estipulado pela equipe de manutenção do laminador é de $95 \%$. Inicialmente, a referência do fabricante foi de $100 \%$, porém com a observação do processo ao longo dos anos, identificou-se a necessidade de controlar este parâmetro em números menores, evitando paradas para redução da temperatura na armadura do motor e visando continuidade operacional.

\subsection{Influência das Variáveis na Força de laminação}

É evidente, ao avaliar o processo, que variáveis tais como largura, temperatura da placa, redução a ser aplicada, composição química do aço, entre outras, têm efeito sobre a força de laminação, que por sua vez afeta o torque que está relacionado com a potência e corrente. Desta forma, para avaliar o IRMS, é necessário que se entenda o efeito das flutuações das variáveis de processo na força de laminação, que por sua vez, afetará o torque necessário para que o processo ocorra e consequentemente, a potência e corrente solicitadas no motor.

A Figura 6 mostra o gráfico da variação da força média de laminação em relação à largura da tira, onde se verificou que quanto maior a largura do material, maior será a 
área de contato com os cilindros de trabalho, resultando no aumento considerável da força aplicada no processo de redução de espessura por meio da laminação.

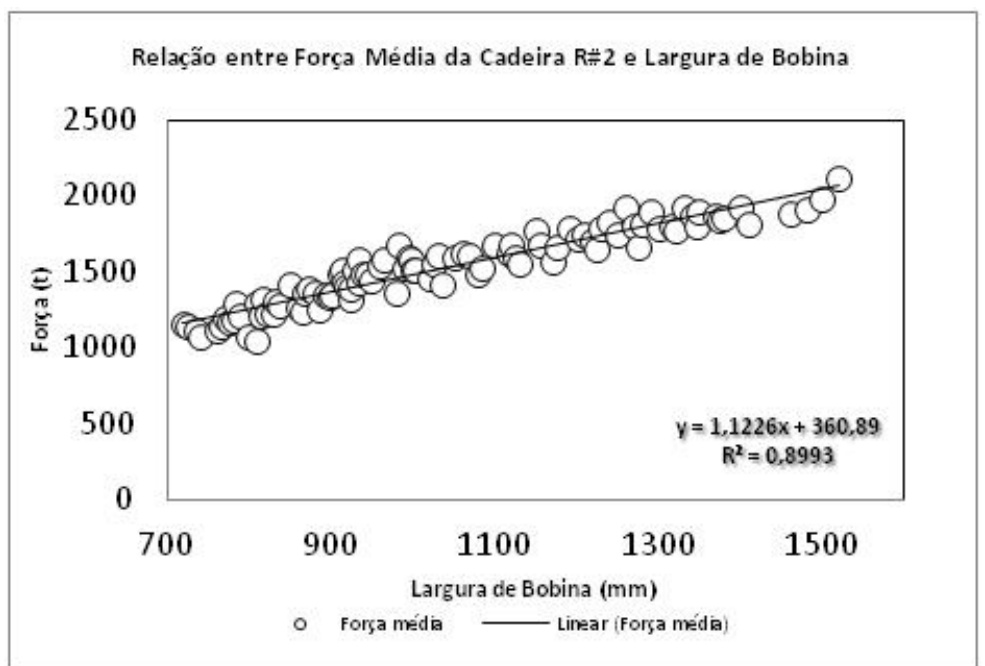

Figura 6: Gráfico de Força Média e Largura da tira para o quinto passe da cadeira reversível. [2]

Não obstante, a Figura 7 apresenta o gráfico de avaliação da força em relação à temperatura de RDT (Rougher Delivery Temperature - Temperatura de entrega do desbaste), que demonstra uma variação decrescente a medida que o processo ocorre com as tiras em temperaturas elevadas de acordo com os parâmetros do processo.

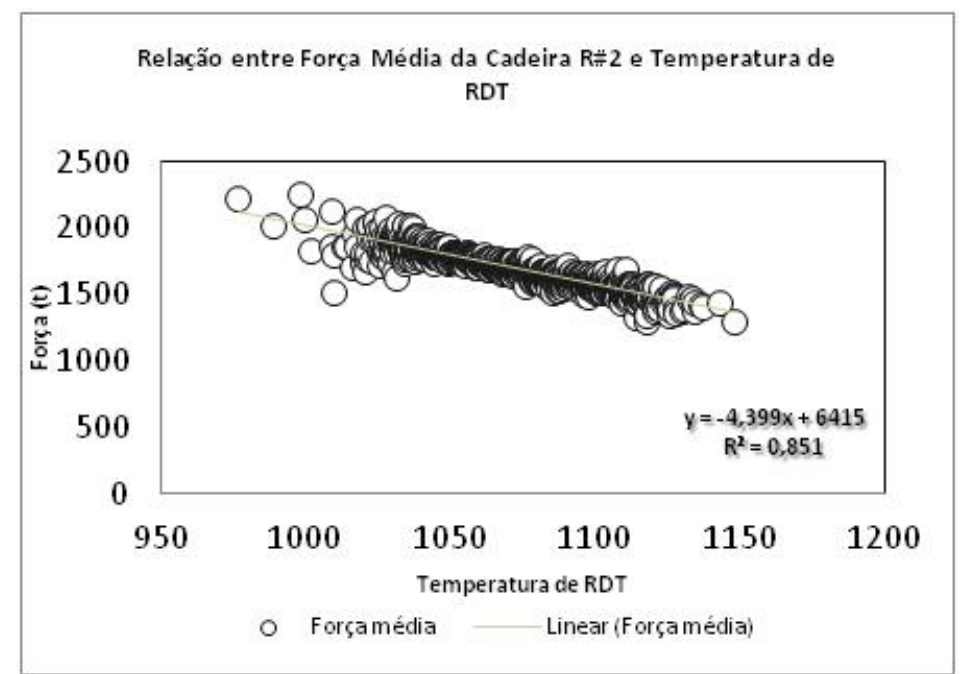

Figura 7: Gráfico de Força Média e Temperatura de RDT para o quinto passe da cadeira reversível. [2]

\subsection{Nova Programação - Modo de Operação RM-NECK}

O modo de operação RM-NECK tem como objetivo aumentar a produtividade da cadeira R2, por meio do incremento de velocidade da mesma, sem que ocorra a degradação dos motores principais da cadeira. A fim de assegurar a preservação do equipamento, a proteção dada aos motores da cadeira R2 é dada pela implantação de diferentes velocidades de laminação, sendo elas variadas de acordo com a composição química do material, espessura e largura da tira. Por meio da teoria das restrições, foram identificados os gargalos de produção para as diferentes espessuras e larguras das tiras laminadas, para que o incremento de velocidade decorresse 
apenas para tiras a serem processadas com o laminador de desbaste como gargalo de produção.

\subsection{Novo mecanismo de incremento de velocidade}

Na modificação do incremento de velocidade, foi-se necessário alterar as lógicas de programação dos Níveis 1 (MELSUCSES) e 2 (SSCL). Os valores atribuídos para o incremento de velocidade foram estabelecidos em testes onde houve a avaliação entre as novas velocidades de trabalho da cadeira R2 e a evolução do IRMS. Os incrementos de velocidade para cada família de material obedeceram ao comportamento do IRMS, ou seja, garantir o trabalho dentro da faixa ótima de estabilidade deste parâmetro. A Figura 8 representa um dos testes realizados para atribuir o melhor valor de incremento de velocidade na cadeira R2 para um material que possui $1200 \mathrm{~mm}$ de largura e grau CSN 2710.

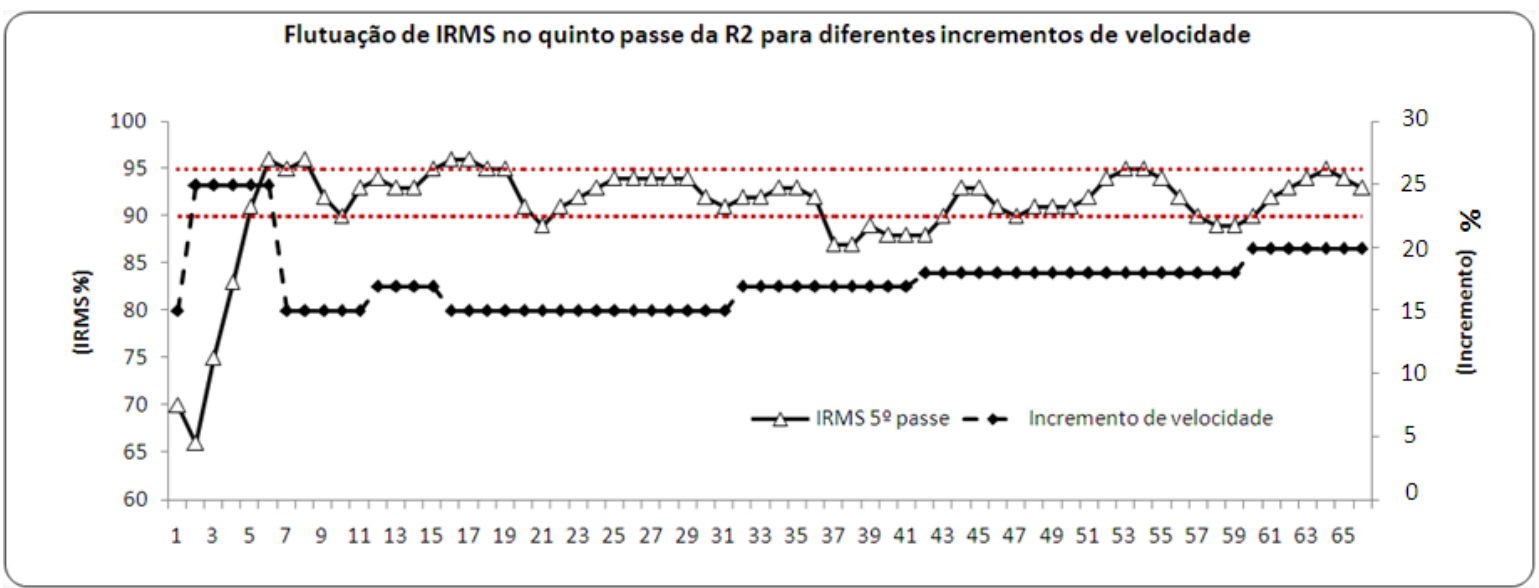

Figura 8: Gráfico de flutuação de IRMS para diferentes incrementos de velocidade. [2]

Para a classe de material usada como exemplo, o valor de incremento de velocidade que demonstrou o melhor aproveitamento do equipamento, mantendo o IRMS dentro do limite de trabalho estabelecido, foi de $15 \%$ a $20 \%$, ou seja, nesta faixa de incremento de velocidade, o IRMS variou na faixa entre $90 \%$ e $95 \%$, conforme limites estipulados pela equipe de manutenção.

\subsection{Modificação no Nível 2 (SSCL)}

A modificação deste nível foi imprescindível para a atualização, dada à necessidade de processamento de um volume maior de informações, a fim de encaminhar apenas os bits ao Nível 1. 
MDLMNT

User name: XXXXX

MANUTENCAO DE ARQUIVOS DO MODELO MATEMATICO (Ver 2.01)

Copyright (C) 2003 CSN MITSUBISH

:MNT: OPEN MRL2F.INC

/ "MRL2F" Aberto com sucesso Tamanho de registro= 640 bytes (

24 simbolos)

/ Este arquivo tem 320000 registros. Selecione as classes

(hierarquia).

1 - 100 Familia de Graus

1 - 8 Classe de Espessura

1 - 8 Classe de Largura

1 - 10 Cadeira Dummy

1 - 5 Reducao de Largura

:MRL2F: LAY 9, 5, 1,1:10,1:5

:MRL2F : SET RMNECK, 1

:MRL2F : SET RMNECK5, 1

Figura 9: Interface para set up do RM NECK no nível 2, para diferentes classes de composição química, espessura, largura, dummy e redução placa bobina. [4]

Por meio do arquivo MRL2F, onde se encontra a classificação das variáveis criadas no projeto, foi possível designar o set up da função RM-NECK para as famílias de aços, espessuras visadas, larguras visadas, condições do equipamento e redução de largura a ser aplicada da placa à bobina. Ao exemplo da Figura 9, o comando LAY 9, $5,1,1: 10,1: 5$ o digito 9 compreende a classe do aço SAE 1006, o digito 5 corresponde a classe de espessuras da tira entre $3,5 \mathrm{~mm}$ e $4,5 \mathrm{~mm}$, o digito 1 corresponde a classe de largura da tira até $800 \mathrm{~mm}$ e os demais dígitos compreendem todas as condições do equipamento e todas as reduções da placa à bobina.

Ainda na Figura 9, a linha do programa: MRL2F: SET RMNECK,1 trata-se do comando responsável por ativar o bit 7 da palavra responsável por encaminhar as informações até o nível 1 e o comando: MRL2F: SET RMNECK5,1 ativa o bit 11 (ou seja, 'B') da mesma palavra satisfazendo as duas condições para que ocorra o incremento de $35 \%$ na velocidade da cadeira de laminação reversível $\mathrm{R} 2$.

Tais informações, necessárias para ativar a função RM NECK, são encaminhadas por meio da palavra de 15 bits da variável DPD2. Esta palavra contém os dados com o padrão de descamação utilizado nos passes da cadeira, porém, os bits 7, 8, 9, 10 ('A'), 11 ('B'), e 12 ('C'), estavam disponíveis e foram utilizados para ativar a nova função.

\subsection{Função RMNECK-DESC}

Durante os testes da função RM-NECK, foi verificado um potencial de ganho de produtividade alterando a distância da placa em relação à cadeira $\mathrm{R} 2$ durante os passes de laminação. Para evitar o risco de manchas de descamação no topo das placas foi necessário elaborar um novo programa no nível 2 para alterar o padrão de descamação para determinados materiais. As placas passariam a parar embaixo da capota de descamação de entrada durante as reversões, este novo padrão foi classificado como Padrão de Descamação RMNECK-DESC. 
Para permitir a entrada da função RMNECK-DESC, foi disponibilizado um novo Bit de comando contido na palavra da variável DPD2 que quando ativado, aciona a função RMNECK-DESC que trabalha juntamente com o modo de operação RM-NECK.

A utilização da função RMNECK-DESC estabeleceu um novo comportamento do IRMS em função da corrente dos motores principais da cadeira R2. O processamento das placas com a função RMNECK-DESC proporcionou uma diminuição do tempo de processamento de placas na cadeira R2 e consequentemente um tempo de espera dos motores inferior ao trabalhado anteriormente, aumentando assim os valores de IRMS. Foi necessário elaborar um novo arranjo de incrementos de velocidade no nível 1 , os percentuais de aceleração para o RMNECK_DESC são menores comparado ao RMNECK, pois, é preciso compensar o tempo menor de cadeira R2 na condição de metal off (sem placa) para o controle do IRMS abaixo de 95\%. Caso por algum problema ou necessidade do processo o operador do Desbaste tenha que alterar o padrão de descamação pela HMI, a função RMNECK_DESC é imediatamente desativada para evitar que as placas fiquem sob a caixa de descarepação.

\section{RESULTADOS}

A implementação do modo de operação RMNECK, juntamente como sua função RMNECK_DESC, apresenta-se como um novo cenário na produção do laminador de tiras a quente. A automação de um modo de operação que visa o aumento de produtividade traz maior eficiência nas reduções de tempo de processamento possibilitando que apenas os materiais que possam apresentar ganhos sejam regidos pelo projeto.

As reduções de tempo de processamento na cadeira $\mathrm{R} 2$ devido ao uso do modo de operação RMNECK apresentaram-se efetivas reduzindo o tempo médio de processamento na cadeira R2 em aproximadamente $4 \mathrm{~s}$. A avaliação dos resultados atrelados à função RMNECK_DESC ainda foi mais satisfatória do que a redução de tempo utilizando apenas o modo de operação RMNECK, a diferença entre as duas etapas do projeto foi de aproximadamente $5 \mathrm{~s}$. A Tabela 2 mostra os tempos de processamento médios na cadeira $\mathrm{R} 2$ para os materiais que foram processados sem a utilização do modo de operação RMNECK, utilizado o RMNECK e por fim os materiais que foram processados com a função RMNECK_DESC. A Tabela 2 também apresenta a diferença entre os tempos de processamento para cada cenário.

Tabela 2: Análise dos tempos de processamento na cadeira R2 para diferentes medos de operação.

\begin{tabular}{c|l|l}
\multicolumn{2}{c}{ Modos de Operação } & $\begin{array}{l}\text { Diferença de } \\
\text { Tempos }\end{array}$ \\
\hline Tempo de Processamento Médio - R\#2 & $79,37 \mathrm{~s}$ & \\
\hline Tempo de Processamento Médio - R\#2 - RMNECK & $75,92 \mathrm{~s}$ & $3,45 \mathrm{~s}$ \\
\hline Tempo de Processamento Médio - R\#2 - RMNECK-DESC & $70,94 \mathrm{~s}$ & $8,43 \mathrm{~s}$ \\
\hline
\end{tabular}

Outro ponto importante a ser analisado, é a resposta dos motores ao uso do modo de operação RMNECK. As Figuras 10 e 11 apresentam os dados das temperaturas atingidas na carcaça e no coletor dos motores inferior e superior da cadeira R2. Os gráficos comparam as temperaturas destes equipamentos a partir de dois cenários: sem a utilização do modo de operação RMNECK; com a utilização do modo RMNECK. 
Avaliação da Temperatura do Motor Superior da R\#2

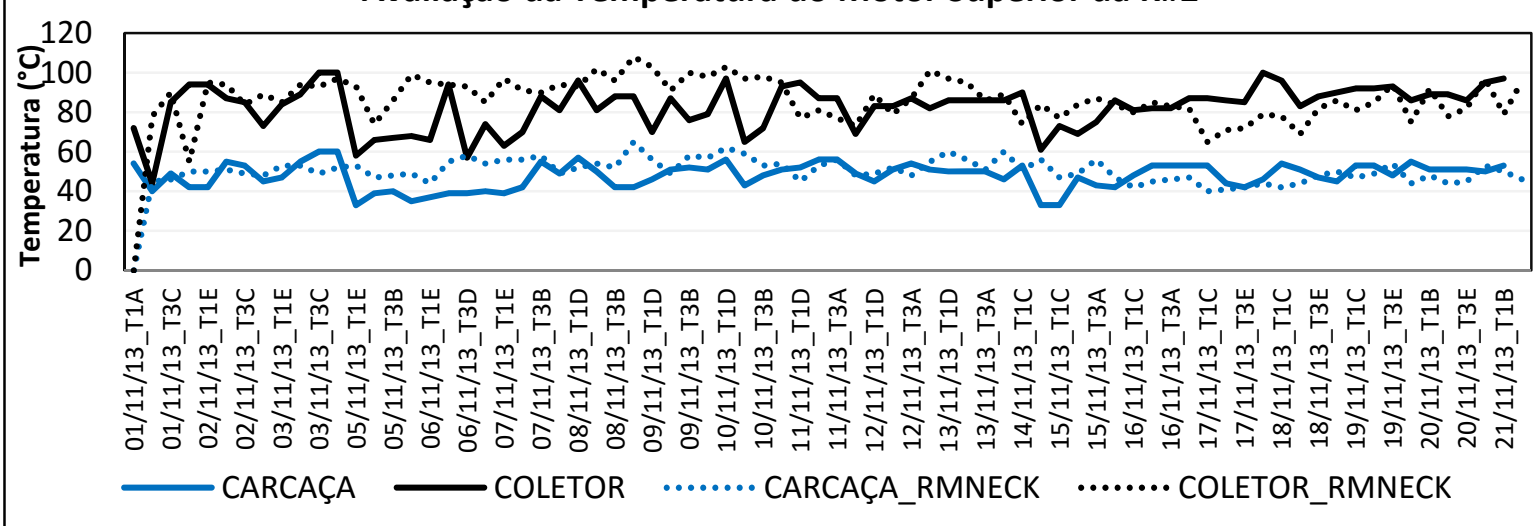

Figura 10: Avaliação da temperatura do motor superior da cadeira R2 a partir da utilização do modo de operação RMNECK.

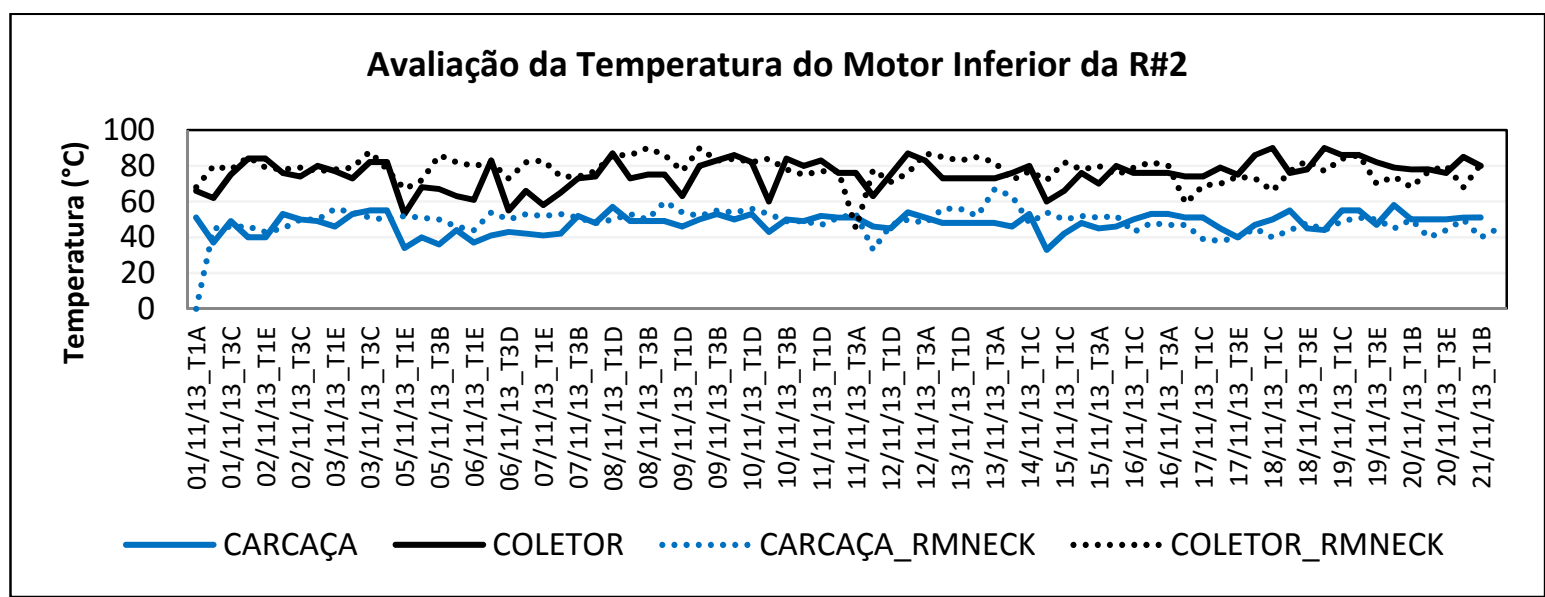

Figura 11: Avaliação da temperatura do motor inferior da cadeira R2 a partir da utilização do modo de operação RMNECK.

Os dados das Figuras 10 e 11 mostram uma estabilidade na temperatura de trabalho do motor da cadeira R2 com a utilização do modo de operação RMNECK. Observase que a temperatura mantém muito próxima da temperatura de trabalho sem a utilização do modo de operação.

\section{CONCLUSÃO}

O modo de operação RMNECK mostrou-se em seis meses ganhos muito significativos. Foram disponibilizadas aproximadamente 47 horas utilizando os modos de operação RMNECK e a função RMNECK_DESC.

As melhores práticas de automação livraram o equipamento da condição em que a velocidade da cadeira era aumentada sem que a produtividade global do laminador de tiras a quente fosse incrementada, o que expunha o equipamento a um desgaste desnecessário. Isto acontecia principalmente para materiais em que o gargalo não era o laminador de desbaste.

A disponibilização de diferentes percentuais de incremento de velocidade possibilitou alcançar aumento de produtividade para materiais em que, com os valores de incremento de $35 \%$ e $45 \%$ do modo de operação anterior ao projeto, levariam o equipamento a uma condição degradada de funcionamento. Um exemplo claro para 
tal situação foi a definição de ganhos na ordem de $7 \%$ para materiais que exigem maiores forças de laminação, como as placas de largura acima de $1350 \mathrm{~mm}$.

Outra vantagem da função RMNECK é o fato de a seleção da função de incremento de velocidade não depender da intervenção do operador, sendo esta decisão uma atribuição da lógica inserida no nível 2, permitindo assim, uma melhor prática no controle do ritmo de laminação.

\section{BIBLIOGRAFIAS}

[1] O. QUELHAS e A. BARCAUI, Teoria da Restrições Aplicada a Gerência de Projetos: Uma introdução à corrente crítica, Laboratório de Tecnologia, Gestão de Negócios e Meio Ambiente, UFF - R. Passo da Pátria, Niterói - RJ.

[2] C. C. NASCIMENTO e R. N. SILVA, Aplicação da automação no aumento de produtividade e melhoria na proteção dos motores principais da cadeira reversível do laminador de desbaste, UBM - 2013, Barra Mansa - RJ.

[3] Control function od furnace entry/delivery, Mitsubishi, 1999.

[4] TechSteel, "Sistema Supervisorio de Controle de Laminação," Volta Redonda, 2012. 